\title{
Gerenciamento de Frequência Escolar Usando Visão Computacional e Redes Neurais Convolucionais
}

\author{
Eder Almeida Chiquito \\ Universidade Positivo \\ Curitiba, Paraná \\ eder.almeida40@gmail.com
Guilherme Eduardo Rodrigues
Universidade Positivo
Curitiba, Paraná
julio10becker@gmail.com

\author{
Guilherme Eduardo Rodrigues \\ Universidade Positivo \\ Curitiba, Paraná \\ olimpio_guilherme@hotmail.com \\ Caroline Mazetto Mendes \\ Universidade Positivo \\ Curitiba, Paraná \\ caroline.mendes@up.edu.br
}

\begin{abstract}
Monitoring students in the classroom plays a key role in the academic follow-up. In addition to attendance control, it also allows studying the impact of absences on learning. In this context, this paper presents the development of a school attendance management system using computer vision techniques and convolutional neural networks. A camera captures images from the classroom. The system then detects the faces of the people in the image, and recognizes students using pre-trained convolutional neural network models. The frequency data generated is stored in the system database and accessed through a web page. In addition, a mobile app has been developed to notify student attendance. The initial results show that the system identifies the registered students and notifies the mobile device of responsible.
\end{abstract}

\section{KEYWORDS}

Frequência Escolar, Monitoramento, Visão Computacional, Redes Neurais Convolucionais

\section{INTRODUÇÃO}

O monitoramento dos alunos em sala de aula tem um papel fundamental no acompanhamento acadêmico. Ausências frequentes e comportamentos inadequados podem causar impacto no aprendizado, e consequentemente, no desempenho acadêmico dos alunos. Assim, além da análise de assiduidade, o monitoramento também possibilita o estudo do comportamento dos alunos em sala de aula.

Como destacado por Suryavanshi e Sankpal [1], a verificação de presença dos alunos normalmente é feita de forma manual pelo professor. Esse método pode não ser viável, dependendo da quantidade de alunos da turma. Além disso, ele não é considerado totalmente assertivo, pois pode ser burlado pelos alunos. Por exemplo, um aluno pode responder a chamada no lugar de outro, ou sair da sala logo após responder a chamada.

Outro problema recorrente em sala de aula é falta de atenção dos alunos [2]. Isso pode ocorrer por variados motivos, tais como o uso de dispositivos eletrônicos, conversa paralela ou hiperatividade e deficit de atenção [3]. Essa situação pode ocasionar baixo desempenho na compreensão dos conteúdos, ocasionando notas baixas e consequente evasão escolar.

Uma maneira de realizar o monitoramento dos alunos é por meio da análise de imagens obtidas por câmeras instaladas dentro das salas de aula. Nessa abordagem, a verificação da presença torna-se mais segura e com menos possibilidades de erros. Além disso, as imagens podem auxiliar os profissionais da educação a detectar quais são os comportamentos de quebra de atenção [4].

Soluções baseadas em análise de imagens têm sido propostas. Por exemplo, para realizar chamada automática e verificação de presença [1,5], detecção de atenção e engajamento [3, 6], incluindo verificação de presença em conjunto com análise de comportamento [7]. Nessas soluções são frequentes o uso de técnicas de visão computacional e redes neurais.

Nesse contexto, este trabalho apresenta o desenvolvimento de um sistema de monitoramento de alunos em sala de aula por meio da análise de imagens, usando visão computacional e redes neurais convolucionais. O sistema verifica os horários de entrada e saída, assim como o tempo em que o aluno esteve presente em sala de aula. A partir dessa plataforma base, o sistema poderá reconhecer os comportamentos dos alunos, com foco nos comportamentos inadequados que podem afetar o processo de aprendizado.

\section{SOLUÇÃO PROPOSTA}

O sistema desenvolvido é composto pelos seguintes módulos: módulo de cadastro; módulo de aquisição; módulo de reconhecimento; e módulo de apresentação dos resultados. O módulo de cadastro consiste em uma aplicação web que permite realizar o cadastro das informações e das imagens das faces dos alunos. Os dados cadastrados são então armazenados na base de dados do sistema.

O módulo de aquisição é composto por uma câmera, sendo este responsável por capturar e enviar as imagens do ambiente da sala de aula para o módulo de reconhecimento. O módulo de reconhecimento, por sua vez, realiza a detecção da presença do aluno em sala de aula. Os dados gerados por esse módulo são armazenados na base de dados do sistema.

O módulo de apresentação dos resultados provê dois meios de acesso aos resultados gerados pelo sistema. O monitoramento dos alunos pode ser realizado por uma aplicação web e por um aplicativo móvel que envia notificações para o dispositivo móvel do responsável.

O método MTCNN (Multitask Cascaded Convolutional Networks) [8] foi usado para realizar a extração de faces das imagens. Esse procedimento é feito em duas situações. Primeiro, o método é usado 
para segmentar as faces das imagens do cadastro, as quais são usadas na etapa de treinamento. Ele também é usado para extrair todas as faces contidas nas imagens obtidas pela câmera em sala de aula, gerando assim imagens com faces individuais para serem comparadas na etapa de classificação. Foi escolhido a utilização desse método pois o mesmo apresentou uma detecção facial extremamente assertiva e otimizada, pois utiliza redes neurais convolucionais em cascata.

Para o treinamento e classificação das imagens foi usado o modelo pré-treinado Facenet [9], que faz uso de redes neurais convolucionais. O método extrai um conjunto de pesos da imagem da face. Assim, os pesos extraídos na classificação são comparados com os pesos extraídos no treinamento usando a técnica de aprendizado de máquina SVM (Support Vector Machine). Caso uma face obtida pela câmera em sala de aula se enquadre nos pesos de uma face treinada, ela é classificada com o rótulo da face treinada.

A detecção de presença ocorre da seguinte maneira. Caso a classificação retorne uma face com pontuação maior que $95 \%$, considerase que o aluno a qual pertence a face está presente em sala de aula. Será atribuída a presença se o aluno for detectado nos primeiros $10 \%$ do tempo de duração da aula. Se não for detectada a presença, ou se esta for detectada após $50 \%$ do tempo de duração da aula, é computada uma falta. Ao fim do processo, os dados de presença são armazenados no banco de dados do sistema.

Os resultados gerados podem ser acessados por meio de uma página web e por um aplicativo móvel. A página web possibilita a visualização da frequência dos alunos por meio de um dashboard. Por fim, o aplicativo desenvolvido gera notificações do tipo: presença detectada, aluno atrasado, aluno ausente e saída antecipada.

Para o desenvolvimento do módulo de reconhecimento foi utilizada a linguagem de programação Python, a biblioteca OpenCV, o framework TensorFlow e o banco de dados PostgreSQL. No desenvolvimento da aplicação web foram usadas as tecnologias Angular.js e Node.js. O aplicativo móvel foi desenvolvido para plataforma Android em conjunto com o Firebase Cloud Messaging (FCM) para gerar as notificações.

\section{CONSIDERAÇÕES FINAIS}

Imagens de alunos de uma turma de um determinado curso de graduação foram coletadas para realização de testes iniciais. Para o treinamento foram utilizadas 20 imagens da face de 43 alunos em diferentes posições. Para diversas imagens com a presença de até 10 alunos, o sistema identificou de forma satisfatória os alunos cadastrados, e também notificou os dispositivos móveis usados nos testes. Contudo, foram observadas falhas na detecção de presença causadas devido a classificações incorretas. Estratégias estão sendo estudadas pra corrigir essas falhas e melhorar o resultado da detecção.

A versão atual do sistema possibilita o monitoramento dos alunos em sala de aula por meio do gerenciamento da frequência. Está em fase de desenvolvimento um algoritmo de reconhecimento de comportamentos que podem causar quebra de atenção. Para isso, foram selecionados dois tipos de comportamentos ( $\mathrm{C} 1$ e $\mathrm{C} 2$ ), de acordo com estudos realizados com psicopedagogos.

Uma nova etapa de aquisição de imagens será realizada. Além de aumentar a base de dados de alunos, será criada uma base de dados de imagens de comportamentos $\mathrm{C} 1$ e C2, obtida por meio de simulações realizadas por alunos em sala de aula. Por fim, o sistema será colocado em execução em uma aula expositiva com duração aproximada de 30 minutos.

O resultado esperado na realização desse projeto é a construção e validação de um sistema de monitoramento de alunos, incluindo gerenciamento de frequência e análise de comportamentos. Esperase que a solução proposta possa auxiliar as instituições de ensino e profissionais da área de educação no monitoramento dos alunos em sala de aula.

\section{REFERÊNCIAS}

[1] SR Suryavanshi and LJ Sankpal. Monitor student's presence in classroom. fournal of Information Technology and Software Engineering, 6(4), 2016.

[2] Ana Laura Godinho Lima. O problema da falta de atenção na escola, 2018. URL https://jornal.usp.br/artigos/o-problema-da-falta-de-atencao-na-escola/. [Acessado em 14 de fevereiro de 2020].

[3] Nigel Bosch. Detecting student engagement: human versus machine. In Proceedings of the 2016 Conference on User Modeling Adaptation and Personalization, pages 317-320. ACM, 2016.

[4] David J Shernoff, Sean Kelly, Stephen M Tonks, Brett Anderson, Robert F Cavanagh, Suparna Sinha, and Beheshteh Abdi. Student engagement as a function of environmental complexity in high school classrooms. Learning and Instruction, 43:52-60, 2016

[5] Shubhobrata Bhattacharya, Gowtham Sandeep Nainala, Prosenjit Das, and Aurobinda Routray. Smart attendance monitoring system (sams): a face recognition based attendance system for classroom environment. In 2018 IEEE 18th International Conference on Advanced Learning Technologies (ICALT), pages 358-360. IEEE, 2018.

[6] Omid Mohamad Nezami, Debbie Richards, and Len Hamey. Semi-supervised detection of student engagement. In PACIS, page 157, 2017.

[7] Jian Han Lim, Eng Yeow Teh, Ming Han Geh, and Chern Hong Lim. Automated classroom monitoring with connected visioning system. In 2017 Asia-Pacific Signal and Information Processing Association Annual Summit and Conference (APSIPA ASC), pages 386-393. IEEE, 2017.

[8] K. Zhang, Z. Zhang, Z. Li, and Y. Qiao. Joint face detection and alignment using multitask cascaded convolutional networks. IEEE Signal Processing Letters, 23(10), 2016.

[9] Florian Schroff, Dmitry Kalenichenko, and James Philbin. Facenet: A unified embedding for face recognition and clustering. In Proceedings of the IEEE conference on computer vision and pattern recognition, pages 815-823, 2015. 